\title{
Olfactory Display Prototype for Presenting and Sensing Authentic and Synthetic Odors
}

\author{
Katri Salminen, Jussi Rantala, \\ Poika Isokoski, \\ Marko Lehtonen \\ School of Information Sciences, \\ University of Tampere \\ firstname.lastname@sis.uta.fi
}

\author{
Philipp Müller, \\ Markus Karjalainen, \\ Jari Väliaho, Anton Kontunen, \\ Ville Nieminen, Joni Leivo, \\ Anca Andreea Telembeci, \\ Jukka Lekkala, Pasi Kallio \\ Biomedical Sciences and Engineering, \\ Tampere University of Technology \\ firstname.lastname@tut.fi
}

\author{
Veikko Surakka \\ School of Information Sciences, \\ University of Tampere \\ veikko.surakka@sis.uta.fi
}

\begin{abstract}
The aim was to study if odors evaporated by an olfactory display prototype can be used to affect participants' cognitive and emotionrelated responses to audio-visual stimuli, and whether the display can benefit from objective measurement of the odors. The results showed that odors and videos had significant effects on participants' responses. For instance, odors increased pleasantness ratings especially when the odor was authentic and the video was congurent with odors. The objective measurement of the odors was shown to be useful. The measurement data was classified with $100 \%$ accuracy removing the need to speculate whether the odor presentation apparatus is working properly.
\end{abstract}

\section{CCS CONCEPTS}

- Human-centered computing $\rightarrow$ Empirical studies in HCI;

\section{KEYWORDS}

Olfaction, multimodal interaction, emotions

\section{ACM Reference Format:}

Katri Salminen, Jussi Rantala, Poika Isokoski,, Marko Lehtonen, Philipp Müller,, Markus Karjalainen,, Jari Väliaho, Anton Kontunen, Ville Nieminen, Joni Leivo, Anca Andreea Telembeci, Jukka Lekkala, Pasi Kallio, and Veikko Surakka. 2018. Olfactory Display Prototype for Presenting and Sensing Authentic and Synthetic Odors. In 2018 International Conference on Multimodal Interaction (ICMI '18), October 16-20, 2018, Boulder, CO, USA. ACM, New York, NY, USA, 5 pages. https://doi.org/10.1145/3242969.3242999

\section{INTRODUCTION}

In recent years, there has been increased interest to add odors to multimodal interaction to complement modalities of vision and hearing. It is easy to envision how the interplay between other senses and odors would enrich user's experiences. Imagine smelling

Permission to make digital or hard copies of all or part of this work for personal or classroom use is granted without fee provided that copies are not made or distributed for profit or commercial advantage and that copies bear this notice and the full citation on the first page. Copyrights for components of this work owned by others than ACM must be honored. Abstracting with credit is permitted. To copy otherwise, or republish, to post on servers or to redistribute to lists, requires prior specific permission and/or a fee. Request permissions from permissions@acm.org.

ICMI '18, October 16-20, 2018, Boulder, CO, USA

(C) 2018 Association for Computing Machinery.

ACM ISBN 978-1-4503-5692-3/18/10 . \$15.00

https://doi.org/10.1145/3242969.3242999 pleasant and refreshing fruity odor while watching a cooking show, alarming scent of smoke while playing a video game, or smelling loved one's body odor during remote communication. Odors have significant effects on evaluating edibility of food, social communication, and learning $[18,26,32]$. Potential multimodal applications vary from health and education to entertainment and media [21]. Odors create significant value to traditional multimodal interaction by creating a stronger feeling of presence in virtual reality [5], affecting positively to dining experience [25], and facilitating odor identification when the audiovisual content is congruent with the odor [14].

Despite this potential, odors are still missing from multimodal interaction. This is because significant methodological challenges related to odor production need to be solved before olfactory displays can be introduced as a mainstream technology [19]. The human perception of an odor is defined by a large number of factors like chemical composition of the odorous substance and its concentration in the air (i.e. intensity) [27]. Most olfactory displays utilize synthesized odors [30]. Synthetic odors aim to reproduce authentic odors consisting of hundreds of chemical compounds by mixing only a few of them $[13,16,27]$. The resemblance between authentic and synthetic odor depends on multiple factors, such as the selected compounds [27]. At the moment, existing olfactory display prototypes $[19,30]$ are designed to accurately evaporate a limited amount of synthetic odorants but the human perception between authentic and synthetic odors in multimodal interaction is not well known. The ability to reduce the number of chemicals while still being able to fool the human senses is a critical issue that deserves much more attention.

One way to study the perception of authentic and synthetic odors in multimodal interaction is focusing on humans' cognitive and emotion-related responses to odor perception. Humans are capable to judge an odor as pleasant or unpleasant [31, 32]. In multimodal contexts, pleasant odors can, for instance, decrease the unpleasantness of a picture [9]. Odors can also elevate the level of arousal [6]. Finally, intensity estimation is a central factor affecting odor perception [10]. For instance, odors can make subjective estimation of pictures more intense [23]. In addition to relying on subjective estimations of odor output, it would be beneficial to equip olfactory displays with odor sensing systems (i.e. electronic noses, eNoses) [20]. This has rarely been done even though eNoses could 
enable objective odor quality verification and intensity estimation $[4,15,17]$.

This paper describes an olfactory display prototype capable of presenting and sensing odors. The prototype was evaluated in an experiment in which authentic and synthetic odors were combined with audiovisual stimulation. The goal was to investigate if participants' cognitive and emotion-related responses differ between authentic and synthetic scents. The task was to watch videos with and without odors. The content of the video was either congruent or incongruent with the odor. The participants were asked if they smelled an odor, what the name of the odor was, and how intense it was. Then, they were instructed to rate the viewing experience by using scales for pleasantness and arousal. The functionality of the olfactory display was also tested with ion-mobility spectrometry (IMS) based eNose [28]. The measured data was classified with a K Nearest Neighbor (KNN) algorithm to compare human and eNose performance in identifying odors.

\section{METHODS}

\subsection{Olfactory Display and IMS}

The basic operating principle of the olfactory display prototype (Fig1a) was to use an air compressor (HBM AS-48) and plastic Teflon ${ }^{\circledR}$ coated tubes to transfer scented air to a mask worn by participants. The air used as carrier gas was dried by pushing it through a cylinder containing silica gel and purified with another cylinder containing activated carbon. The air pressure was then lowered to $1 \mathrm{bar}$. An airflow of $1.4 \mathrm{l} / \mathrm{min}$ went into a tube connected to the cap of a flask. The flask contained the lemon peel (authentic odor). The odorous components of the lemon peel evaporated in the flask, forming a headspace (Fig1b). Another tube connected to the cap of the flask carried the odorized air out of the flask. The odor was presented by opening a manual valve directing air flow through the flask. For brevity, the authentic lemon odor vented from the flask is referred to as flask in the remainder of this paper.

For creating the synthetic scent, $1.4 \mathrm{l} / \mathrm{min}$ of air flow was divided between three evaporation units where limonene (synthetic odor) was evaporated using ceramic heating elements. Airflow was calibrated with Sensidyne's Gilian Gilibrator-2 NIOSH to match the airflow coming out of the flask. Limonene was pumped to each of the three heating elements by using a separate syringe pump. The rate of evaporation was controlled by adjusting the speed of the pumps and the voltages of the heating elements. The pumping speeds and heating elements were controlled using Matlab R2015b running on a Lenovo laptop PC (Windows 7 Enterprise, 64bit Operating System). Outward tubes after the evaporation units were partly covered with Omega rope heaters to achieve stable air temperatures of $35-36{ }^{\circ} \mathrm{C}$. This was done to prevent premature condensation of limonene in the tubes. Temperatures and output of limonene odor were monitored with IMS, ChemPro 100i (Fig1c, [28]).

To achieve controlled diffusion of the odors, participant was fitted with a mask (Ecolite Adult mask with $50 \%$ venture valve) covering both nostrils and nasal area. The odors were presented to the participant by connecting the tube of the mask to the outlet of either the flask or olfactory display. When no odor was presented, the valves were closed and the outlet was connected to an activated

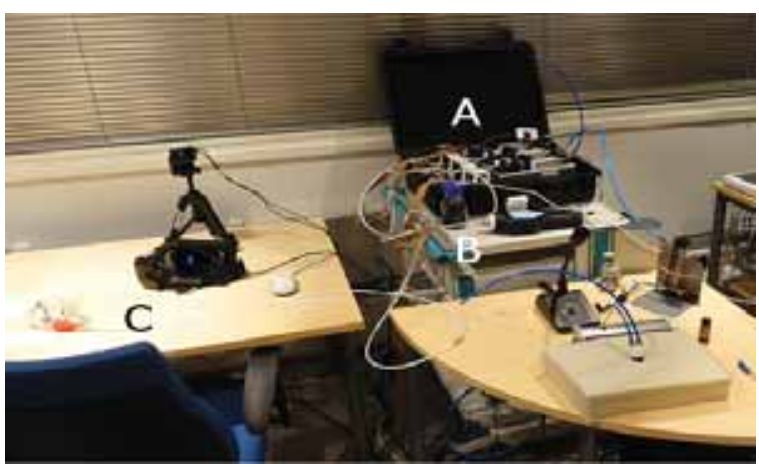

Figure 1: Figure 1: Experimental setup: olfactory display (a), ChemPro100i and (c) Oculus Rift + mask.

carbon cylinder that absorbed the odor. An Oculus Rift DK2 headset was worn on top of the Ecolite mask. It allowed to display video stimuli to the participant and block the view to the tubing, which would have revealed the source of the odors.

\subsection{Odors}

We used lemon peel as the authentic odor and limonene as the synthetic odor. Lemon peel was chosen because it is easy to synthesize. The authentic odor is composed of up to $97.4 \%$ limonene [8], which is described to have an odor of lemon [24]. Thus, the differences between the selected authentic and synthetic odors was expected to be small. We used $97 \%$ limonene (CAS number 5989-275) from Sigma Aldrich ${ }^{\circledR}$. The three pumps of the olfactory display pumped undiluted limonene with pumping speed of $150 \mu$ l per hour and heating voltage of $1.6 \mathrm{~V}$. For the authentic odor, $5 \mathrm{ml}$ of freshly grated lemon peel stored at a room temperature was used.

\subsection{Human Tests}

2.3.1 Participants. A total of 29 voluntary participants took part in the study (14 males, 3 smokers, mean age 33.4 years, range 19 58 years). All the participants reported to have normal or corrected to normal vision and sense of smell and no oversensitivity to odors or allergies. The participants were informed about the purpose of the study and they signed a consent form. The study protocol was approved by the Ethics Committee of the Tampere region.

2.3.2 Videos. Three videos were chosen. Two of them were congruent with the odors (i.e. lemon spray and lemon picking) and one was incongruent (i.e. potato peeling). The lemon spray video was 21 seconds long, and visualized a kitchen hack in which a sprayer was attached to a fresh lemon, and lemon juice was sprayed [1]. The lemon picking video was 23 seconds long, and visualized a person picking off lemons from a tree [2]. The potato peeling video was 32 seconds long, and visualized a gimmick in which a potato was boiled, and then peeled with fingers [3]. In all videos a male voice narrated the events in English

2.3.3 Procedure. A trial proceeded as follows. A video was presented to the participant via virtual reality glasses. Simultaneously, an outlet from either flask to present lemon peel or from evaporation units to present limonene was connected to the tube attached 
to the mask. When the video was presented without an odor, the outlet of the flask was connected to the tube, but the manual valve was kept closed so that the participant smelled only room air. This was done to mimic potential tactile perception of an outlet being attached to the mask. After watching a video, the participant was asked "did you smell an odor" ("yes" or "no"). If the participant reported to smell an odor, the second task was to freely name the odor in question. Then, they were asked to rate the intensity of the odor and pleasantness and arousal of the viewing experience on three nine-point bipolar scales that varied from -4 (mild, unpleasant or calm) to +4 (strong, pleasant or aroused). All videos and odor conditions were presented in fully randomized order making a total of nine different stimuli. Conducting the experiment took approximately 40 minutes.

2.3.4 Data Analysis. ARTool [29] was used to do align rank transformation for the non-parametric rating data. Then, a two-way within-subjects (odor $\times$ video) repeated measures analysis of variance (ANOVA) was conducted. In a case of statistically significant interaction effect, simple main effect analysis for odor and video were conducted separately. Pairwise t-tests with Bonferroni corrected $\mathrm{p}$-values were used for post hoc tests.

\subsection{IMS Measurements}

2.4.1 Procedure. Limonene and lemon peel were measured five times for five minutes with ChemPro 100i. The presentation of an odor to the ChemPro 100i was similar to presenting an odor to a participant. The outlet from either olfactory display or flask was connected to a tube attached to ChemPro $100 \mathrm{i}$ to get the IMS readings. Each IMS reading consists of 14 measurements; 7 for the positive and 7 for the negative channels. ChemPro-UIP v1.3.3.3 software was used to save the data. For presenting the odor from flask to ChemPro $100 \mathrm{i}$ a pressurized headspace concentrating odorous molecules was formed. To exclude the potential differences between limonene and lemon peel presentation style or intensity to the results, one more measurement condition was added. In this condition, $5 \mathrm{ml}$ of lemon peel was placed on a plate located $3 \mathrm{~cm}$ from the ChemPro 100i sensor.

2.4.2 KNN classification of the IMS data. A KNN classifier [12] was used to analyze IMS samples. It works in real time and needs no re-training when new odors are added to the database. This is important in early work such as this where training data accumulates as new odors are introduced to the system. The basic idea behind the KNN approach is to compare a 14-dimensional IMS sample (i.e., $\mathrm{x}(\mathrm{us})=[\mathrm{x}(\mathrm{us}) 1 \ldots \mathrm{x}(\mathrm{us})])$ of an unlabeled odor with labeled training samples stored in a database, find the $\mathrm{K}$ training samples closest to $\mathrm{x}(\mathrm{us})$, where closeness is measured by the Euclidean distance, and label this odor based on the labels of the $\mathrm{K}$ closest training samples using a majority vote. The training database contained measurements of limonene, lemon peel from flask, and lemon peel from table. The aim was to determine the misclassification rates (e.g. how often the odor was classified as lemon peel when it was limonene).

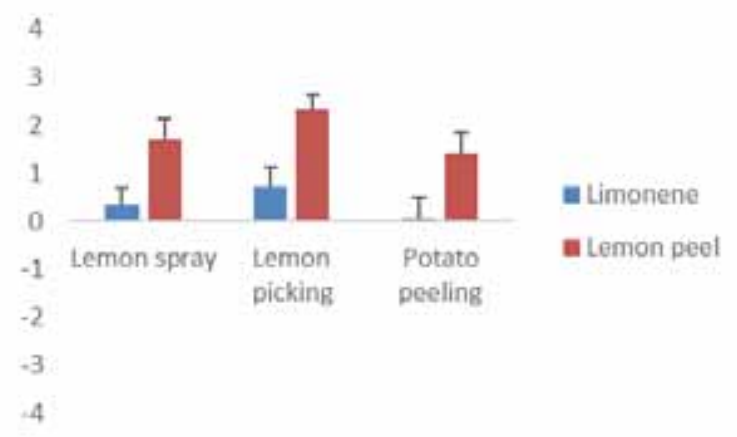

Figure 2: Figure 2: Means and standard error of the means (SEMs) for the ratings of the intensity.

\section{RESULTS}

\subsection{IMS Data}

KNN-based classification of IMS readings with $\mathrm{K}=3$ and exhaustive search yielded misclassification rates of $0 \%$ for all odors. Using k-dimensional tree search [7] instead of exhaustive search did not change the misclassification rates but approximately halved the classification time.

\subsection{Human Data}

3.2.1 Odor recognition and naming. All the participants always reported to smell lemon peel. When the odor was limonene, 27 participants reported to smell the odor when the video was lemon spray or potato peeling and 28 when it was lemon picking. 2 participants reported to smell the odor while watching lemon peel and 1 while watching lemon picking videos in no odor condition. All the participants always named lemon peel as lemon-like. 14 participants named limonene as lemon-like when the video was lemon spray or lemon picking and 9 when it was potato peeling.

3.2.2 Subjective ratings. For the ratings of intensity, a $2 \times 3$ (odor $\times$ video) ANOVA showed a statistically significant main effects for odor $\mathrm{F}(1,28)=32.7, p<0.001$ and video $\mathrm{F}(2,56)=5.2, p<0.05$ (Fig2). Post hoc comparisons showed that the participants rated viewing experience more intensive when the odor was lemon peel than when it was limonene ( $\mathrm{md}=1.4, p<0.001$ ), and that they rated the viewing experience more intensive when the video was lemon picking than when it was potato peeling $(\mathrm{md}=0.8, p<0.01)$.

For the ratings of pleasantness, a $3 \times 3$ (odor $\times$ video) ANOVA showed a statistically significant interaction of the main effects $\mathrm{F}(4,112)=6.5, p<0.001$ (Fig3). The simple main effects for both odor $\mathrm{F}(2,56)=17.3, p<0.001$ and video $\mathrm{F}(2,56)=3.8, p<0.05$ were statistically significant. Post hoc comparisons for odor showed that the participants rated viewing experience more pleasant during smelling of lemon peel than limonene when the video was lemon spray $(\mathrm{md}=1.2, p<0.001)$, lemon picking $(\mathrm{md}=1.6, p<0.001)$, or potato peeling ( $\mathrm{md}=0.7, p<0.05$ ). The participants also rated viewing experience during smelling of lemon peel more pleasant than no odor condition when the video was lemon spray ( $\mathrm{md}=$ $1.4, p<0.001$ ) or lemon picking ( $\mathrm{md}=1.1, p<0.001$ ). Finally, the participants rated no odor condition as more pleasant than limonene 


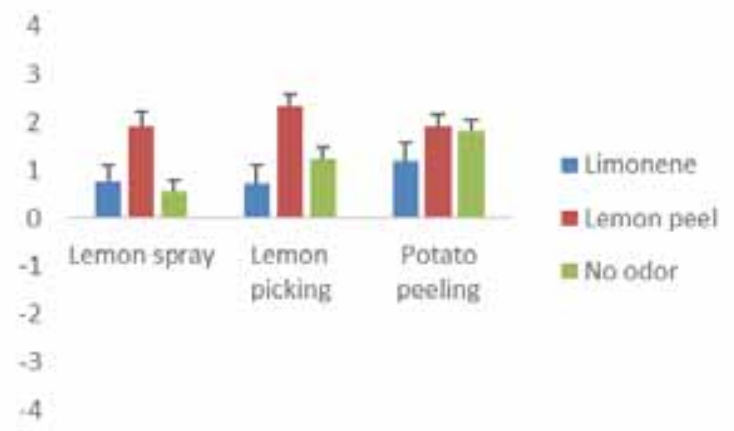

Figure 3: Figure 3: Means and SEMs for the ratings of the pleasantness.

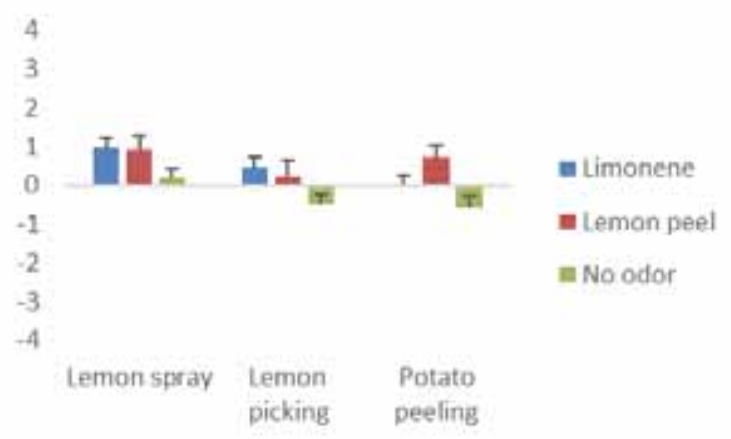

Figure 4: Figure 4: Means and SEMs for the ratings of the arousal.

when the video was potato peeling ( $\mathrm{md}=0.7, p<0.05$ ). Post hoc comparisons for video were not statistically significant.

For the ratings of arousal, a $3 \times 3$ (odor $\times$ video) ANOVA showed a statistically significant main effects for odor $\mathrm{F}(2,56)=11.0, p<$ 0.001 and video $\mathrm{F}(2,56)=4.9, p<0.05$ (Fig4). Post hoc comparisons showed that the participants rated viewing experience less arousing when there was no odor than when the participants smelled lemon peel $(\mathrm{md}=0.6, p<0.01)$ or limonene $(\mathrm{md}=0.7, p<0.01)$. Participants also rated the viewing experience of lemon spray video as more arousing than lemon picking $(\mathrm{md}=0.6, p<0.05$ ) or potato peeling $(\mathrm{md}=0.6, p<0.05)$.

\section{DISCUSSION}

The results showed that the participants rated lemon peel as more intense than limonene. This was supported by the data where the participants reported if they smelled an odor. Only lemon peel was smelled in $100 \%$ of the cases. Limonene was detected almost equally well suggesting that almost all of the participants were able to smell the odor. As expected based on previous studies [23], odors were rated as more intense when the video was congruent with the odor. Surprisingly, only half of the participants named synthetic limonene odor as lemon-like, even though previous studies [14,24] indicate that limonene smells like lemon and input from other modalities further facilitates the naming of odors towards the content of the video. Odor perception is affected by age, sex, societal and cultural factors, memory, and experience [14]. Our analyses indicated that the age and sex of participants did not significantly influence the naming and societal and cultural backgrounds of the participants were homogenous. A possible factor explaining the differences is earlier experience of the limonene or similar odors. Many of the participants who did not name limonene as lemon-like associated the odor with cleaning solvents. It is feasible that earlier experience of the odor as a component of household cleaning liquids affected the naming of limonene in our study.

The fluctuation in the naming of limonene between participants and intensity ratings between odors highlights the need for odor output measurement. We tested this idea. Data measured with IMS and classified with KNN showed $100 \%$ accuracy in identifying the odors. The classifier was able to label the odor correctly based on its authenticity and intensity. This means that the information was available for the humans as well, but their sensory systems were not sufficiently precise for detecting and labeling the odors perfectly. The result confirms the need for objective measurement of the output of an olfactory display to support the unreliable human nose. In future, IMS readings can be transferred to an olfactory display, which can then adapt the output based on the measurements. For instance, intensity can be modified or odor changed in real time.

Viewing experience with odors was always rated as pleasant. Rating was affected by odor authenticity and video congruency. Lemon peel odor resulted in higher ratings of pleasantness than limonene, suggesting that authentic, complex odors are more efficient in evoking pleasant experiences during multimodal interaction. This effect was rather surprising. Limonene is reported to smell like lemon [24] and pleasant [22]. In addition, lemon peel was rated as more intense than limonene, and there is a tendency to rate more intense odors as unpleasant [11]. The discussed issues related to odor naming may affect the current finding. Similarly to previous studies [23], participants tended to rate viewing experience as more pleasant with congruent than incongruent videos. Furthermore, the viewing experience was rated as more arousing with odors than without odors. This result is in line with previous studies [6]. As the authenticity of the odor or the congruency between video and odor did not affect the ratings. This suggests that any odorant should elevate the level of arousal during multimodal interaction.

Taken together, we have built and tested an olfactory display equipped with IMS for multimodal interaction. We were able to show that viewing experience while smelling authentic or synthetic odors was evaluated positively in terms of pleasantness and arousal. For the most pleasant experience the odor needed to be authentic and content of the video congruent suggesting that careful selection of odorant and presentation context is important. Next, we aim to study the effects of a wider set of authentic and synthetic odors in virtual reality and continue developing methods for odor measurement, classification, and reproduction.

\section{ACKNOWLEDGMENTS}

This research was funded by the Academy of Finland (grant numbers 295432, 295433 and 295434) and the Finnish Cultural Foundation. 


\section{REFERENCES}

[1] 2018. Retrieved June 15, 2018 from https://www.youtube.com/watch?v= FJvNWYkHNg4\&t=68

[2] 2018. Retrieved June 15, 2018 from https://www.youtube.com/watch?v= jnQA7NPuFB

[3] 2018. Retrieved June 15, 2018 from https://www.youtube.com/watch?v= $8 \mathrm{cCX710BEyU}$

[4] S Ampuero and JO Bosset. 2003. The electronic nose applied to dairy products: a review. Sensors and Actuators B: Chemical 94, 1 (2003), 1-12.

[5] Oliver Baus and Stéphane Bouchard. 2017. Exposure to an Unpleasant Odour Increases the Sense of Presence in Virtual Reality. Virtual Real. 21, 2 (June 2017), 59-74. https://doi.org/10.1007/s10055-016-0299-3

[6] Moustafa Bensafi, Catherine Rouby, Vincent Farget, Bernard Bertrand, Michel Vigouroux, and André Holley. 2002. Autonomic nervous system responses to odours: the role of pleasantness and arousal. Chemical Senses 27, 8 (2002), 703709.

[7] Jon Louis Bentley. 1975. Multidimensional binary search trees used for associative searching. Commun. ACM 18, 9 (1975), 509-517.

[8] Gracia P Blanch and Graeme J Nicholson. 1998. Determination of the enantiomeric composition of limonene and limonene-1, 2-epoxide in lemon peel by multidimensional gas chromatography with flame-ionization detection and selected ion monitoring mass spectrometry. Journal of chromatographic science 36, 1 (1998), 37-43.

[9] Marius Hans Braun, Gilang Andi Pradana, George Buchanan, Adrian David Cheok, Carlos Velasco, Charles Spence, Andoni Luis Aduriz, Jade Gross, and Dani Lasa. 2016. Emotional priming of digital images through mobile telesmell and virtual food. International fournal of Food Design 1, 1 (2016), 29-45.

[10] Sylvain Delplanque, Didier Grandjean, Christelle Chrea, Laurence Aymard, Isabelle Cayeux, Benedicte Le Calve, Maria Ines Velazco, Klaus R Scherer, and David Sander. 2008. Emotional processing of odors: evidence for a nonlinear relation between pleasantness and familiarity evaluations. Chemical Senses 33, 5 (2008), 469-479.

[11] Richard L Doty. 1975. An examination of relationships between the pleasantness, intensity, and concentration of 10 odorous stimuli. Perception \& Psychophysics 17, 5 (1975), 492-496

[12] Richard O Duda, Peter E Hart, David G Stork, et al. 2001. Pattern classification. 2nd. Edition. New York 55 (2001)

[13] A Escudero, B Gogorza, MA Melus, N Ortin, J Cacho, and V Ferreira. 2004. Characterization of the aroma of a wine from Maccabeo. Key role played by compounds with low odor activity values. Fournal of Agricultural and Food Chemistry 52, 11 (2004), 3516-3524.

[14] Gheorghita Ghinea and Oluwakemi A. Ademoye. 2011. Olfaction-enhanced Multimedia: Perspectives and Challenges. Multimedia Tools Appl. 55, 3 (Dec. 2011), 601-626. https://doi.org/10.1007/s11042-010-0581-4

[15] Guillaume Hudon, Christophe Guy, and Jacques Hermia. 2000. Measurement of odor intensity by an electronic nose. Fournal of the Air \& Waste Management Association 50, 10 (2000), 1750-1758.

[16] Elodie Le Berre, Thierry Thomas-Danguin, Noelle Beno, Gerard Coureaud, Patrick Etievant, and John Prescott. 2007. Perceptual processing strategy and exposure influence the perception of odor mixtures. Chemical senses 33, 2 (2007), 193-199.

[17] Amy Loutfi, Silvia Coradeschi, Ganesh Kumar Mani, Prabakaran Shankar, and John Bosco Balaguru Rayappan. 2015. Electronic noses for food quality: A review. fournal of Food Engineering 144 (2015), 103-111.

[18] Maureen Morrin and S Ratneshwar. 2000. The Impact of Ambient Scent on Evaluation, Attention, and Memory for Familiar and Unfamiliar Brands. Fournal of Business Research 49, 2 (2000), 157 - 165. https://doi.org/10.1016/S0148-2963(99) 00006-5

[19] Takamichi Nakamoto. 2016. Essentials of machine olfaction and taste. John Wiley $\&$ Sons.

[20] Takamichi Nakamoto. 2016. Olfactory display and odor recorder. Essentials of machine olfaction and taste. Wiley, Singapore (2016), 247-314.

[21] Niall Murray Niall, Brian Lee, Yuansong Qiao, and Gabriel-Miro Muntean. 2016. Olfaction-Enhanced Multimedia: A Survey of Application Domains, Displays, and Research Challenges. ACM Comput. Surv. 48, 4, Article 56 (May 2016), 34 pages. https://doi.org/10.1145/2816454

[22] AC Paillard, Maryam Lamôré, Olivier Etard, J-L Millot, L Jacquot, P Denise, and G Quarck. 2014. Is there a relationship between odors and motion sickness? Neuroscience letters 566 (2014), 326-330.

[23] Nobuyuki Sakai, Sumio Imada, Sachiko Saito, Tatsu Kobayakawa, and Yuichi Deguchi. 2005. The effect of visual images on perception of odors. Chemical Senses 30, suppl_1 (2005), i244-i245.

[24] Sigma-Aldrich ${ }^{\circledR}$. 2018. Ingredients Catalogue for Flavors and Fragrances. Retrieved June 15, 2018 from http://go.sigmaaldrich.com/FFCatalogPDF

[25] Charles Spence and Jozef Youssef. 2015. Olfactory dining: designing for the dominant sense. Flavour 4, 1 (14 Nov 2015), 32. https://doi.org/10.1186/ s13411-015-0042-0

[26] Richard J Stevenson. 2009. An initial evaluation of the functions of human olfaction. Chemical senses 35, 1 (2009), 3-20.

[27] Thierry Thomas-Danguin, Charlotte Sinding, Sébastien Romagny, Fouzia El Mountassir, Boriana Atanasova, Elodie Le Berre, Anne-Marie Le Bon, and Gérard Coureaud. 2014. The perception of odor objects in everyday life: a review on the processing of odor mixtures. Frontiers in psychology 5 (2014), 504.

[28] Mikko Utriainen, Esko Kärpänoja, and Heikki Paakkanen. 2003. Combining miniaturized ion mobility spectrometer and metal oxide gas sensor for the fast detection of toxic chemical vapors. Sensors and Actuators B: Chemical 93, 1-3 (2003), 17-24.

[29] Jacob O Wobbrock, Leah Findlater, Darren Gergle, and James J Higgins. 2011. The aligned rank transform for nonparametric factorial analyses using only anova procedures. In Proceedings of the SIGCHI conference on human factors in computing systems. ACM, 143-146.

[30] Yasuyuki Yanagida and Akira Tomono. 2013. Basics for olfactory display. In Human Olfactory Displays and Interfaces: Odor Sensing and Presentation. IGI Global, 60-85.

[31] Yaara Yeshurun and Noam Sobel. 2010. An odor is not worth a thousand words: from multidimensional odors to unidimensional odor objects. Annual review of psychology 61 (2010), 219-241.

[32] Manuel Zarzo. 2008. Psychologic dimensions in the perception of everyday odors: pleasantness and edibility. Fournal of Sensory Studies 23, 3 (2008), 354-376. 